\title{
MRAE Americas
}

\section{Report of the EVI Working Session DRAFT}

\author{
Prepared for the \\ Massachusetts Ocean Partnership
}

November 19, 2009

MRAG Americas, Inc.

65 Eastern Avenue, Unit B2C

Essex, MA 01929

wwW.mragamericas.com 


\section{Table of Contents}

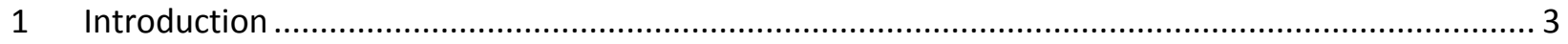

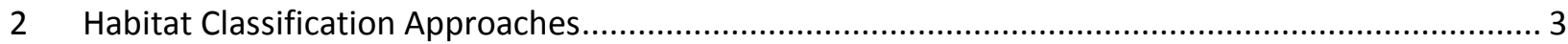

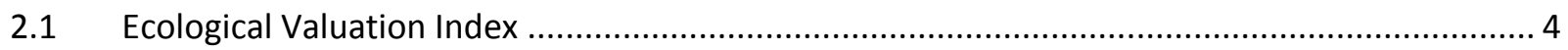

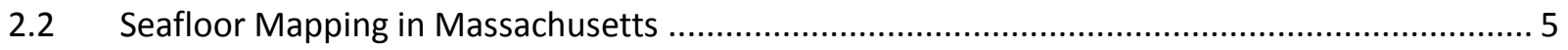

2.3 Ecologically and Biologically Significant Areas on Eastern Scotian Shelf ................................ 6

2.4 Cumulative Impact of Human Activities on Massachusetts Marine Ecosystems...................... 8

2.5 Ecological Risk Assessment and Productivity and Susceptibility Analysis............................... 9

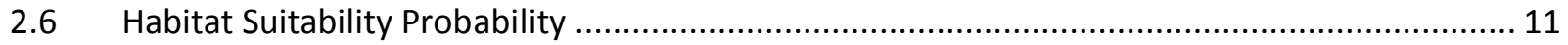

2.7 Ecosystem Service Trade-off Analysis .............................................................................. 13

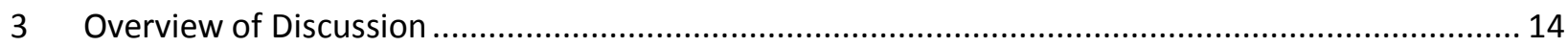

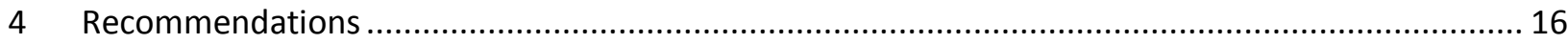

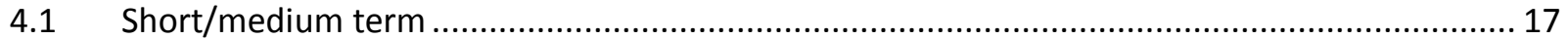

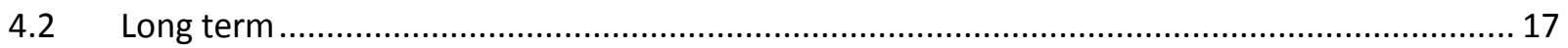




\section{Introduction}

The Massachusetts Oceans Act of 2008 set forth 15 principles to guide development of the Massachusetts Ocean Management Plan, including to "identify and protect special, sensitive or unique estuarine and marine life and habitats; value biodiversity and ecosystem health; and respect the interdependence of ecosystems." Based on these three principles, the Commonwealth of Massachusetts' Executive Office of Energy and Environmental Affairs (EEA) was charged with developing a tool to identify special, sensitive, or unique estuarine and marine life and habitats (SSU) while considering biodiversity and ecosystem health. The process resulted in the development of an ecological valuation process that utilizes spatial analysis techniques to systematically evaluate the ecology of Massachusetts waters; this Ecological Valuation Index (EVI) utilized available data to contribute to the identification of SSU areas. The EVI methodology considered vast quantities of data and provided a means for ecological valuation of areas; however, the methodology was limited to biotic data and did not include abiotic or socio-economic measures. Furthermore, there were considerable limitations in available data. Based on the limitations of the EVI, the EEA needed to consider alternative tools that could enhance or replace EVI as a management tool for use in development of the Ocean Management Plan.

In July, 2009, the Massachusetts Ocean Partnership (MOP) asked MRAG Americas to review the Massachusetts' EVI and to provide advice and options for enhancing the methodology in the short (summer and fall 2009) and long term. MRAG Americas, MOP, and EEA convened a working session in October 2009 to review the existing EVI methodology and to develop recommendations for possible enhancements to the EVI. Working session participants included invited scientists, members of the State of MA Science Advisory Council, members of the EVI work groups (comprised of representatives from other state government offices, e.g. DMF, CZM), MRAG Americas, and MOP. The working group met for one full day at UMASS, Boston; several participants attended remotely. This report provides an overview of the tools presented at the working session, the discussion that ensued among participants and the resulting recommendations. The recommendations provided are largely conceptual, given the limited time participants had to further clarify them. These recommendations will likely require further discussions, particularly among the involved parties and developers of the tools, to expand the concepts into operational recommendations.

The working session was guided by the following goals:

- Make recommendations on the appropriate role(s) of EVI as a management or decision support tool in the context of other science, data, and decision support tool advancements

- Develop recommendations on possible enhancements to the EVI methodology

\section{Habitat Classification Approaches}

A selection of available approaches to the problem of classifying and valuing marine habitats was presented, including the EVI methodology developed during the drafting of the Massachusetts Ocean Plan. This section summarizes the approaches and notes their limitations. 


\subsection{Ecological Valuation Index}

\subsubsection{Summary}

The EVI was developed in response to the challenges of defining and identifying SSU areas in the MA Oceans Act Management Planning Area $(0.3-3 \mathrm{~nm})$. Along with these primary challenges, Stateappointed work groups identified limitations of available data for species and habitats, issues with data quality, limitations of ecosystem structure and function knowledge, and complications in creating a system that would allow for comparisons between different species and habitats. These gaps and limitations furthered the need for development of a suitable tool that would utilize available data and attempt to evaluate the ecological importance of species and habitats.

The development process proceeded with creating a tool to "measure" the ecological value of various areas based on biotic data. The EVI work group considered approaches used elsewhere to identify and classify marine ecosystems, including the approach by the Department of Fisheries and Oceans Canada (DFO) to identify Ecologically and Biologically Significant Areas (ESBAs). The work group also considered refinements to generate an overall biological value for areas of the ocean and to create Biological Valuation Maps (BVMs) such as those developed for the Belgium part of the North Sea (BPNS). Classification of value was generally based on the goals and objectives of the overall management effort.

The EVI work group developed an Ecological Valuation Protocol based on the concepts of the BPNS approach and further refined it to assess the intrinsic significance of biotic locations within the planning area. Considerations in development of the protocol included ecological characteristics in Massachusetts state waters and for species that have limited spatial distribution, are endangered and/or threatened, or are of global and/or regional importance. The resulting protocol excluded abiotic features and socio-economic implications.

The protocol used available data for individual species to indicate their perceived ecological importance using certain criteria: major contributions to fitness, spatial rarity, and populations of global and/or regional importance. The endangered or threatened status of a species (roseate terns, right whales, humpback whales, and fin whales) was not considered a direct contributor to ecological importance but treated separately in the identification of SSU areas. All criteria were given a binary presence/absence score and equal weighting. The scores were generated for each species dataset and the sum for all datasets at a $250 \times 250 \mathrm{~m}$ grid cell resolution. Species were grouped by taxa, and the range of scores was sorted into quartiles (high, med-high, med-low, low). Final scores were unit-less.

\subsubsection{Limitations}

The EVI was intended to serve two major needs for the State Management Plan: identifying areas for specific activities and categorizing SSU areas to guide planning of activities and to reduce impacts in Massachusetts coastal waters. However, given data limitations, the protocol was unable to fully meet these needs.

Limitations identified by the EVI work group included:

- Inconsistencies in data availability, spatial resolution and data quality 
- Absence of abiotic data in the protocol

- Limitations in available data for describing ecosystem value

- Difficulties incorporating new data as understanding of ocean ecosystems evolves

These limitations were apparent in spite of the fact that the species data used in the EVI were among the most complete data sets and covered the species that managers and society are usually most concerned with, including fish, mollusks, crustaceans, seabirds, and marine mammals.

In general, the EVI served well to identify fitness on a broad scale, but it lacked spatial contrast necessary for disaggregating areas with the largest fitness contribution into smaller regions of highest value. The SSU areas that resulted from the valuation process were largely driven by fish species due to higher data availability (and that fact that each data series was equally weighted). The EVI is a good species driven tool to relate fitness to areas and to identify co-occurrence of species; however, the design resulted in a method that was driven by data rich species and lacked an abiotic component. Ultimately, the EVI was not used in the existing science framework, and members of the work group expressed that it would be difficult for EVI to address issues arising from regime shifts including climate change.

\subsection{Seafloor Mapping in Massachusetts}

\subsubsection{Summary}

The Massachusetts Office of Coastal Zone Management (CZM) has initiated a Seafloor Mapping Program to better understand seafloor geology and biology and to aid in balancing the impacts of human activity with the protection of coastal and marine resources. The ability to map important ocean habitats and identify areas for protection may address needs identified in the Massachusetts Ocean Management Plan. The draft Ocean Plan (2009) has identified areas suitable for protection from impacts associated with human development and outlined additional work needed to further identify and characterize such areas, beginning with mapping. The Seafloor Mapping Program is a long term effort by CZM to map and classify the wide variety of seafloor habitat types within the Commonwealth's waters. Additionally, benthic habitat mapping is specifically identified in the science framework action plan.

"Benthic habitat mapping and classification, which includes identifying the spatial extent of the ocean planning area's existing habitats and - to the extent feasible, their usage by flora and fauna - is a fundamental requirement for effective ocean planning" (Science Framework Action Plan, 2009).

CZM has a cooperative initiative with the United States Geologic Survey (USGS) to collect seafloor topography and surficial geology data to produce high resolution maps; these maps will provide a foundation to characterize seafloor habitats and inform ocean planning. The seafloor mapping being conducted is geologic mapping to characterize the surface and subsurface geologic framework offshore of Massachusetts. The long-term goal is to produce digital, high-resolution, geologic (surface and subsurface) maps that will serve the needs of research, management and the public.

This is the first effort to map the Massachusetts seafloor, and it will greatly inform decision making. As of September 2009, about one-third of Massachusetts' waters have been mapped. Completed areas 
include Nahant to Gloucester, Boston Harbor, Cape Ann to Salisbury, Hull to Duxbury, Northern Cape Cod Bay, and Eastern Cape Cod; current areas being mapped are Buzzards Bay and Vineyard Sound. The effort aims to map potential habitat; therefore, a classification system is needed to further guide the mapping efforts. The mapping program has selected the habitat framework developed by Green et al. (2009), which has been formally adopted by and incorporated into the Coastal and Marine Ecological Classification Standard (CMECS) developed by NOAA in cooperation with NatureServe. The framework integrates water column to sub-benthos components. Although this framework is currently in draft form, it may become a national standard. To date, habitat classification has not been initiated on the maps developed. Initial work with CMES has resulted in 84 unique potential habitat code combinations, although scale was not fully considered when adding attributes and few geologic attributes were used. All oceanographic parameters used came from the Finite-Volume Coastal Ocean (FVCOM) model developed by UMASS and WHOI.

\subsubsection{Limitations}

There are presently several limitations in the effort to map and classify seafloor habitat within Massachusetts' waters and work is underway to address them. There remains to be a clear definition of the minimum mapping unit ( $\mathrm{MMU}$ ) that can be accurately mapped. The determining factors include availability of data, refinement of sediment classification, incorporation of additional abiotic parameters (i.e. temperature; salinity; stratification; sea-surface height; tidal, wind, and density driven current; bottom stress; and potentially others), and an overall assessment of accuracy (thematic and spatial). A classification framework has been accepted, though mapped areas have not yet been classified.

The seafloor mapping effort is driven more by abiotic data than fitness of biotic factors; this is nearly an opposite approach to that used by the EVI. It will be beneficial to management to determine a process to bring these two approaches together so that areas identified can be assigned value. It is important to note that the hierarchal scale will change, as not all species in the EVI (e.g. seabirds) are dependent on seafloor habitat. In addition, the habitat mapping efforts can be conducted at a much finer scale than the resolution of most of the biological data. The difficulty of bridging different scales of biotic and abiotic data was illustrated in the BNPS approach. However, a classification system fitted to the maps will allow for adjustments in scale that complement biological data. The working session discussed a potential process where the EVI assesses value (the "sensitive" of SSU), and the maps identify spatial scale (the "areas"). The need remains for identifying vulnerability, which can be plausibly achieved through several approaches.

\subsection{Ecologically and Biologically Significant Areas on Eastern Scotian Shelf}

\subsubsection{Summary}

The Eastern Scotian Shelf Integrated Management (ESSIM) Initiative was developed in response to Canada's Oceans Act of 1997. This initiative established the first Ecosystem-Approach to Management (EAM) of Canada's three oceans. The ESSIM Initiative is a collaborative ocean management and planning process being led and facilitated by Fisheries and Oceans Canada (DFO) within an EAM framework. DFO has identified five Large Ocean Management Areas (LOMAs) as the geographical basis for EAM, the first 
of which was ESSIM. The primary aim of ESSIM is to develop and implement an Integrated Ocean Management Plan for this large marine region.

DFO has drafted a national plan to conserve sensitive habitat in each LOMA. Seven marine protected areas (MPAs) have already been established with six additional MPAs to be implemented by 2012. In order to achieve this, DFO has identified a national suite of ecosystem objectives for EAM, identified which of these relate to MPAs within ESSIM, and initiated an MPA implementation process. The ecosystem objectives (developed in a 2001 national workshop) address biodiversity, productivity, and habitat considerations, and have been adopted under the Healthy Ecosystems goal of ESSIM. The MPArelated objectives of ESSIM require the protection of representative habitat and ecologically distinctive or significant areas.

DFO has undertaken considerable work over the past decade to identify and map ecological priority areas in the ESSIM area. This includes a benthic community classification approach developed by V. Kostylev and based upon the theoretical work of R. Southwood. This approach considers the benthic community's scope for growth (i.e. productivity) and the natural disturbance that it experiences, measured by a number of readily available attributes. It provides a theoretical basis for the distribution of benthic organisms and a measure of their biological resilience. DFO has also developed criteria to identify ecologically and biologically significant areas (EBSA); these areas are differentiated as being those "which are particularly important or significant with regard to specific ecosystem structural or functional properties from all other areas where the structure may occur or the function may be served, but which are not justifiably designated as significant" (DFO, 2004). Primary EBSA criteria include uniqueness (unique, rare, distinct characteristics), aggregation (life history context), and fitness (influence on survival \& reproduction), and are supplemented by secondary criteria, including resilience (ability of area to respond to impact) and naturalness (pristine versus disturbed).

A 2006 DFO workshop identified 27 offshore and 36 inshore EBSAs while a 2008 working group, using MARXAN $^{1}$, assessed how well the offshore EBSAs aligned with the MPA objectives of ESSIM. This resulted in a map of ecological priority areas which were then presented to the broader ESSIM stakeholder community. This map was then used to identify three Areas of Interest (AOI) for ESSIM (Middle Bank, Misaine Bank/Eastern Shoal and St. Anns Bank) as the first step in the MPA creation process. These AOls were selected through determining: (1) which ecological priorities areas best matched the ESSIM MPA goals and (2) which areas already received protection to human impacts. If areas were already protected, they were not chosen as an AOI. The AOI selection process involved discussions with the stakeholder community. Consultation is currently underway with the ESSIM

\footnotetext{
${ }^{1}$ MARXAN is a decision support tool to identify alternative zoning configurations that minimize social, economic and ecological cost using a specific set of performance criteria. It has been used extensively in marine and coastal planning to develop multiple-use zoning plans, including MPAs that balance the varied interests of stakeholders. (Ball, I. R., and H. P. Possingham. 2000. MARXAN (V1.8.2): Marine Reserve Design Using Spatially Explicit Annealing, a Manual pdf (267KB))
} 
community, to be completed by November 30, 2009, on which AOI should be recommended as an MPA. Once the intended MPA is announced, the MPA designation process, involving articulation of specific conservation objectives, boundaries, regulations, etc., will commence, with final designation by 2012.

\subsubsection{Limitations}

The ESSIM highlights the need for overall goals to guide management and illustrates how implementation efforts can realize these goals. In the application described here, the focus was to identify marine protected areas rather than to contrast areas within the broader ecosystem as a planning tool for a range of coastal ocean activities. The Canadian exercise focused on identification of priority areas for protection based upon both conservation requirements and the practical realities of implementation. As well, ESBAs consider the spatial distribution of biological attributes such as fitness and measure an area's influence on the fitness of an organism. This was not done in the EVI. However, the applicability of this approach for deciding between the relative impacts of management alternatives has yet to be tested.

\subsection{Cumulative Impact of Human Activities on Massachusetts Marine Ecosystems}

\subsubsection{Summary}

The Massachusetts Ocean Partnership (MOP) contracted scientists at the National Center for Ecological Analysis and Synthesis (NCEAS) to develop maps of the impact of human activities in Massachusetts Marine Ecosystems. This work follows the methodology of Halpern et al. (2008). The study aimed to identify and understand how human activities are affecting the marine ecosystems of New England from the intertidal zone out to the boundary of the US exclusive economic zone, 200nm offshore, with a particular focus on the waters off Massachusetts.

This work is presently in draft form; initial data collection (Round 1) has been completed and provided valuable information for the development of the draft Ocean Management Plan. The availability and quality of data and expert survey sample sizes from Round 1 were not sufficient to provide robust and meaningful results; therefore, Round 2 is currently filling data gaps and is anticipated to be completed by the end of 2009 .

One of the primary aims of this work is to provide information on the spatial pattern of activities and their overlap, and assess the impacts of single and multiple activities on ecosystems. The work builds on the habitat maps developed by CZM (described above). The approach entails combining maps of stressors and ecosystems and applying ecosystem vulnerability weights to those maps to obtain vulnerability scores, as judged by expert opinion through survey. To estimate the direct and indirect ecological consequences of human activities, ecological experts with experience in the region were surveyed to quantify the vulnerability of each of the different marine ecosystem types in the region (e.g., eelgrass, hard bottom continental shelf, rocky intertidal) to each of 55 different current and emerging anthropogenic drivers of change. Ecosystem vulnerability is based on 5 criteria: spatial scale, frequency, trophic impact, percentage change (i.e. resistance), and recovery time (resilience of an ecosystem after a disturbance). Available spatial data were gathered and in some cases modeled for the distribution of a subset of those human activities across the study region. This resulted in maps for 20 
different activities in categories like fishing, climate change, and pollution. These maps were combined with maps of the distribution of 15 distinct marine ecosystems, broken into 3 categories (intertidal, subtidal coastal, offshore). Cumulative impact scores were achieved using a weighted model and were calculated for every $1 \mathrm{~km}^{2}$ pixel of the ocean based on the impact of an activity and the ecosystem vulnerability score. The resulting cumulative impact map was developed by overlaying the 20 threat maps onto the ecosystems, and using the vulnerability scores to translate the threats into a metric of average cumulative ecological impact.

\subsubsection{Limitations}

In general, this novel approach is ecosystem-based and ecologically grounded, providing a framework that is scalable from local to global. It allows data to be added easily and sets a baseline for monitoring future changes.

Mapping of cumulative impacts in Massachusetts waters provides a valuable link to habitat distribution and ecosystem vulnerability. However, this work is presently in draft form, with an additional survey currently in process to fill information gaps, particularly in ecosytem-by-activity vulnerability weights. The cumulative impact scores developed from the approach are driven by ecosystem vulnerability weights; therefore, accurate and complete ecosystem habitat maps are needed. This approach follows from the work being conducted by CZM. Additionally, species level data are not included in this approach. Links between human activities and ocean health are done at the ecosystem level, which presents an opportunity to introduce aspects of the EVI to this approach.

\subsection{Ecological Risk Assessment and Productivity and Susceptibility Analysis}

\subsubsection{Summary}

The Australian Commonwealth Scientific Industrial Research Organization (CSIRO) and Fisheries Management Authority (AFMA) developed an ecological risk management (ERM) framework as a key step in their implementation of ecosystem-based fisheries management (EBFM) ${ }^{2}$. The framework provides for a precautionary evaluation of vulnerability of an ecological component (species, habitat, or community) and uses a hierarchal approach that builds with data availability. The method was designed to assess the impact of fishing activities on an ecological component, but has valuable application beyond fishing activities.

The ERM framework uses three levels of assessment ranging from qualitative to quantitative. Level 1 (Scale Intensity Consequence Analysis, SICA) is a qualitative analysis to identify effect of activity on the whole component considered. Level 2 (Productivity Susceptibility Analysis, PSA) is a semi-quantitative analysis to identify effect on units within the component considered. Level 3 is a fully quantitative assessment that considers temporal and spatial variation in the unit. The framework was designed to be precautionary in the absence of data and can serve as an efficient screening and prioritizing tool that allows comparisons (e.g. between fisheries, habitats). Using a set of prescribed attributes to measure

\footnotetext{
2 Hobday, A. J., A. Smith, H. Webb, R. Daley, S. Wayte, C. Bulman, J. Dowdney, A. Williams, M. Sporcic, J. Dambacher, M. Fuller, T. Walker. 2007. Ecological Risk Assessment for the Effects of Fishing: Methodology. Report R04/1072 for the Australian Fisheries Management Authority, Canberra.
} 
vulnerability of an ecological component, the framework assesses the risk that an ecosystem will change beyond acceptable limits if ecological objectives are not achieved.

The approach designed for the ERAEF can assess risk to 5 different ecological components (target species; byproduct and bycatch species; threatened, endangered, and protected species (TEP); habitats; and communities (including food chains)), which collectively define an ecosystem. The risk assessment approach applies to habitats, in particular in that a habitat represents the unit in which species interact. The matrix includes essential ecological processes which sustain ecosystem health. Loss or impairment of habitat integrity results in direct impacts to species, communities, and ecosystem structure and function. To assess the habitat risk of a range of activities associated with fishing, AFMA and the Marine Stewardship Council (MSC) ${ }^{3}$ have developed a habitat-specific methodology.

The methodology is composed of several steps:

- Habitat Scoping, which involves identifying the habitat units ('types') occurring within the jurisdictional boundary of the fishery, primarily on the basis of digital image data. Benthic habitats are classified according to biological and physical elements. Scoping generates a list of habitat types per fishery area.

- A Level 1 (SICA) analysis, which is typically conducted to identify which activities lead to a significant impact on an ecological unit. This analysis considers the effects of an activity on whole component and is used as a rapid screening tool to eliminate low risk elements (either activities or components) from further analysis. Level 1 allows for a qualitative comparison of habitat types in an area.

- A Level $2^{4}$ Productivity Susceptibility Analysis, which is built on assumption that risk to an ecological unit (species, habitat, community) from a given activity will depend on the unit's susceptibility or "exposure" to the fishing activity and the unit's productivity, which determines the rate at which the unit can recover after depletion or damage by the fishing activity. Components or activities determined moderate/high risk during the SICA require further assessment at Level 2. The PSA approach is structured around a set of attributes that describe the resilience and intrinsic risk of each particular unit to impacts.

- Overall Risk or Vulnerability Scores are calculated through a combination of individual attribute scores (a measurement of habitat vulnerability is based on 11 attributes, derived from expert opinion). The approach is precautionary in assigning a high risk score in the absence of data. Individual scores for productivity and susceptibility are plotted on a PSA chart to yield an overall risk score.

\footnotetext{
${ }^{3}$ Hobday, A. J., and A. D. M. Smith. 2008. MSC risk-based framework: technical document September, 2008. Marine Stewardship Council, London.

${ }^{4}$ MRAG Americas has conducted over 150 PSAs for federal fishery stocks to assess vulnerability as it relates to setting Annual Catch Limits.
} 


\subsubsection{Limitations}

This approach, particularly the PSA, is a powerful tool to evaluate vulnerability of units to fishing activities. PSA evaluates the relative ecological risk without abundance estimates using an ordinal scale. However, given a three or five point scale, which is usual, it is unclear if there would be sufficient contrast between adjacent areas to meets the needs of the Ocean Plan.

In addition to the cumulative impacts works described above, PSA may serve to assess vulnerability of areas or species to activities. The approach was designed specifically to assess fishing activities, yet it can be altered to address a suite of activities. Potential applications for the approach include scoring species (or habitats) not currently included in the EVI or specific habitat types and locations in Massachusetts state waters; providing baseline comparisons between habitat types or species with varying levels of available information; and confirming or modifying findings from the EVI or other tools. Evaluation of habitats complements the spatial component needs identified by the working session participants. Conceptually, habitat vulnerabilities can be scored and mapped out spatially. As noted, however, this work has not been conducted for Massachusetts waters and the focus of the approach is on fishing activities. A substantial effort would be required to adapt the methodology to the broad range of activities under management in the Ocean Plan.

\subsection{Habitat Suitability Probability}

\subsubsection{Summary}

As required by the Magnuson-Stevens Fishery Conservation and Management Act (MSA), scientists at the National Marine Fisheries Service (NMFS) and MRAG Americas, Inc. worked to develop a modeling approach for identifying essential fish habitat (EFH) to assess the likely importance of habitats for each species and life stage (called Habitat Suitability Probability - HSP). The approach was developed in context of the West Coastal EFH Environmental Impact Statement (EIS). This work was done by evaluating the probability that particular habitats are suitable for particular species and life stages. The groupings of all the HSP scores are combined to make a Habitat Suitability Index (HSI). The HSI relates to EVI in identification of essential habitat for species; it could also be modified to incorporate both nonfishery species and criteria that allow evaluation of issues beyond EFH.

Data fall into the following major categories:

- Physical Habitat - a Geographical Information System (GIS) containing bottom type, location, bathymetry, latitude, and other structural considerations

- Fish Distributions and habitat preferences - from NMFS survey data, distribution information, life history and habitat use literature reviews

- Fishing Effects - a GIS for spatial fishing information, a review of gear operations, and a review of habitat sensitivity to gear

- Non-fishing Effects - a GIS for spatial information, a review of non-fishing activities, and a review of habitat sensitivity to these activities

- Existing Habitat Protection - a GIS with a Marine Protected Area inventories and other regulatory (e.g. closed) areas 
The value of habitats as EFH is then estimated using a Bayesian Network (BN) model that takes information about the preferences of species/life stages for certain habitat conditions and uses this to plot habitat suitability probabilities by species and life stage, where possible, across the habitats mapped in the GIS. The probability that a habitat polygon is of a particular substrate type, depth range, and latitude is calculated based on the uncertainty in the data. For each substrate type, a habitat suitability score is available. Hence the final probability of a parcel being suitable for a given species or life stage is the combination of the expected habitat characteristics and the suitability score for those characteristics. The identification of EFH can then proceed based on a policy decision regarding the threshold probability above which the polygon is identified as EFH and below which it is not. In addition to identifying EFH, the federal regulations require identifying Habitat Areas of Particular Concern (HAPC).

The following considerations guide the designation of HAPCs:

- Importance of the ecological function provided by the habitat

- Sensitivity to human-induced environmental degradation

- Stress on habitat from development activities

- Rarity of the habitat type

The BN model that was developed calculates a score, quartile (or similar measure for each of the four considerations), and relative risk to habitat. It is relatively easy to use the model to determine HAPC by measuring whether a characteristic exceeds a management threshold, or if the overall relative risk exceeds a management threshold.

A BN model risk assessment can also isolate areas of vulnerability and adverse impact. The model will assess the impacts on EFH and the extent to which the fishery and the ecological function of habitat can be enhanced by changing anthropogenic activities (e.g. use of certain fishing gears, such as mobile and fixed gears). The output of the risk assessment is presented in a GIS format to facilitate spatial consideration of management alternatives. The main data sources for this model are:

- Sensitivity of habitats to contact from fishing gears

- Rates of habitat recovery from gear impacts

- Historical fishing effort (amount and frequency) by area

- Non-fishing impacts

\subsubsection{Limitations}

The work conducted resulted in habitat suitability probabilities for 82 species of Pacific groundfish at their various life stages. The adult output was the strongest due to information availability. The work to identify HAPCs resulted in the designation of MPA conservation areas to protect Pacific Coast groundfish habitat. In order to keep the information relevant, NMFS is charged with reassigning the HSPs every 5 years. Performing an HSI is a longer term effort, particularly depending on the modifications required. This approach can provide useful information on habitat utilization by species. However, it is focused on protecting individual groups of species rather than addressing alternative management approaches. 
While the methodology could be adapted to activities other than fishing, the data requirements and model effort needed are substantial.

\subsection{Ecosystem Service Trade-off Analysis}

\subsubsection{Summary}

The Massachusetts Ocean Partnership (MOP) contracted a group of scientists at the National Center for Ecological Analysis and Synthesis (NCEAS) and Boston University to conduct a spatially-explicit ecosystem service trade-off analysis. Addressing trade-offs among stakeholders and user-groups is critical for making management decisions, because activities regularly co-occur and interact, in turn affecting services provided by the ecosystem. The approach for the analysis is under development and work has only recently begun. This section will briefly review the conceptual approach and subsequent plan of work.

The approach for analyzing trade-offs among ecosystem-services is based on simple economic decision theory. To be effective and useful to management, the method needs to focus on delivery of services, explicitly look at trade-offs, and be applicable to management decisions. The approach is designed to determine how and where management can optimize the delivery of ecosystem services being considered. This approach models the effect of a given management action on the delivery of services at a particular location and the resulting value provided to society. Trade-offs are then assessed between pairs or sets of services based on the given action. By exploring the consequences of all possible management actions, one can identify which actions produce unnecessary tradeoffs (i.e. one could increase the amount of one or both services of interest at no extra cost by shifting to a different management option) and what the set of best actions is. The set of best options lies along an 'optimal frontier.' Choosing among the optimal solutions is then a social or political decision.

Trade-off analysis is a valuable management tool because it will be able to elucidate where initially presumed trade-offs do not exist, and it can demonstrate the cost of single-sector management when trade-offs do exist. Trade-off analysis also reveals inferior management options, identifies a set of optimal management options, addresses any number of services and therefore allows for comprehensive assessments, does not require services to be measured in dollars, makes decisions and their consequences explicit, and allows direct evaluation of candidate services for different locations (e.g. in Massachusetts waters).

The project will take a two-pronged coordinated and collaborative approach to trade-off analysis: a simpler technique with fewer details being conducted in the short-term by NCEAS and a longer, more detailed analysis by Dr. Les Kaufman (Boston University). Together the research teams will identify key trade-offs and sites to analyze, derive "optimal frontier" solutions, and map scenarios for the chosen 
locations. The results will be plugged into a management decision tool known as MIDAS ${ }^{5}$ (Marine Integrated Decision Analysis System), which will serve as a visualization tool for stakeholders.

\subsubsection{Limitations}

Trade-off analysis will be critical for managers as the Ocean Management Plan is further developed and revised in the future. However, the work on this project is in progress, and is not yet available for application. This approach will not explicitly identify areas for activities and/or protection, but it will aid decision-makers in deciding where different activities should be allowed to occur in MA waters.

\section{Overview of Discussion}

Following presentation of the various approaches, the workshop discussed the State's requirements for spatial ocean management and the appropriate tool(s) to meet those needs. The collection of work presented shares the goals of identifying and protecting essential habitat, although the parameters defining identification and protection differ. Additionally, the methods use different starting points. This section summarizes participant discussions and recommendations on the best tools for habitat classification and evaluation to support the Massachusetts Oceans Act.

For specific management reasons, the State needs to discriminate between areas of importance. The development of the Ecological Valuation Index approached this mandate with a species focus by relating fitness to areas and co-occurrence of species. This approach is important for particular species, given that those highlighted in the method are typically the species of concern for managers and the public. The Seafloor Mapping Program initiated by CZM was designed to meet the requirements of the Science Framework, to answer a call for better understanding of seafloor geology and biology, and to aid in balancing the impacts of human activity with the protection of coastal and marine resources. This approach uses habitat and abiotic data as its starting point, fulfilling the need for spatial information, and is able to use data at a finer scale than the data on the species included in the EVI. Though the mapping effort is making impressive strides to map benthic habitats in Massachusetts waters, it does not assign value of those habitats but rather identifies habitat type by location. It also does not consider data quality.

The approach of Halpern et al. aims to map cumulative impacts of human activities on certain habitat types. This analysis can address multiple activities and provides an evaluation of vulnerability, but it lacks a species focus. Data quality is addressed through the expert survey. In context of the Massachusetts Ocean Management Plan, the Halpern et al. approach will become more useful when it includes the concept of ecological value.

\footnotetext{
${ }^{5}$ MIDAS is a spatial decision support system software designed to support the process of decision-making for managers of marine management areas (MMAs) and policy makers. This tool is designed to assist the various users to understand the critical determining factors (CDFs) for the success of an MMA so that they can plan accordingly. The tool is designed to estimate the likely effects of MMAs based on ecological, socioeconomic, and governance conditions.

http://www.conservation.org/Documents/Marine Integrated_Decision_Analysis System User Guide.pdf; http://people.bu.edu/suchi/midas/index.html
} 
The PSA approach has the ability to identify species and/or habitat vulnerability but is designed to focus on fishing activities and may be difficult to adapt to multiple activities. For the purposes of the Massachusetts Oceans Plan, the identification of vulnerability is very useful but may need to be approached by different means.

The HSI design does focus on species and their habitat requirements and also has a fisheries focus. It cannot, however, account for multiple activity impacts though the model does use a computational approach to address data quality. Identification of HAPCs using this approach does address the value of identified areas. Conducting HSPs for a variety of individual species would also be challenging and time consuming. How the results would be used in the specific context of the Massachusetts Oceans Plan is not clear.

The ecosystem service trade-off modeling is a valuable approach for classifying and valuing habitat for the comparison of proposed management options. However, this modeling effort is not fully developed and will be driven by much of the data needed for habitat mapping and human impacts mapping. Once fully developed, it will fill an important need for management planning.

To operationally satisfy the Oceans Act principles identified at the start of this report, the management tool(s) chosen need to address spatial components, species, and impacts in order to determine if one location is more important than another with respect to ecological valuation. This means that the methodology needs to incorporate enough information so that there is real contrast between areas (e.g. the areas have a range of values, rather than everything being estimated as of high value).

Federal and state laws already provide identification and protection for high priority areas (i.e. eelgrass and right whale habitat) and acknowledge their value. The state needs a tool that will help managers determine where different activities should be allowed to occur, considering ecological and socioeconomic factors. Development of the appropriate tool will be difficult, particularly in light of scaling issues. Most of the approaches presented use data at different scales, and in terms of biological considerations, some valuable species have different habitat requirements over their life history. The following is a list of considerations for selecting the appropriate tool for the state to achieve its objectives:

- Ecological and socioeconomic factors are included in a way that is clear to managers and the public

- Biotic and abiotic factors are included as well as measures of vulnerability

- There is a consistent, statistically valid approach to resolving scaling issues between data sets and approaches (e.g., species data is often collected at different spatial and temporal resolution than abiotic data)

- The methodology improves the ability to discriminate between areas and the impacts of uses in an area in with respect to the goals of the Act

- The methodology improves the ability to identify areas for greater levels of protection (including fully protected reserves)

- Habitats are classified with respect to a finite set of attributes to focus data collection efforts 
- The approach can easily, consistently, and transparently incorporate new information as it becomes available

This list of considerations will likely involve a selection of tools, and decision makers should not narrow their attention to one tool. It is probable that a combination of tools will be needed to sufficiently advise managers. There was general consensus that EVI as it presently exists is not the right tool to meet the needs of the state, nor will it be the defining tool with further development. EVI does a good job at bringing together some information on primary species, but it falls short in adequate consideration of habitats and biodiversity.

The participants agreed that there needs to be a linkage between biotic and abiotic components along with some measure of vulnerability. Seafloor mapping overlaid with impacts of human activities can provide a base to such an approach but lacks a valuation process. Overall, the right tool for this effort must accommodate the managers' objectives, work within the context of management, and be repeatable.

\section{Recommendations}

The EEA is presently working to finalize the draft Management Plan by December 31, 2009, and may consider the following recommendations as the science framework of the plan is further developed. The working group's recommendations are intended to provide the conceptual framework and initial steps needed to further the existing tools for management application.

In the development of these recommendations, participants considered the practicality of a management tool and what advances could reasonably be accomplished in the coming months. EVI is an informative method but not the right stand-alone tool or starting point to identify SSUs. EVI will be helpful for comparison with a broader approach, incorporating habitat classification and cumulative impacts analysis. Conceptually, the working group recommended that the identification of SSUs should proceed from physical characteristics of the ecosystem to ecological characterization, vulnerability to impacts, and then valuation. Habitat mapping is a logical first step, including mapping of pelagic habitat, which presents some difficulties but can be approached through the oceanographic model. Benthic and pelagic habitat characterization will have to proceed iteratively as new information is acquired and can be incorporated.

The working group suggested the following approach:

1. Seafloor mapping and classification work should serve as the basis of the management tool.

2. Human Impact Mapping is the appropriate framework for discriminating between areas in order to evaluate alternative proposals for activities in the management area.

3. The EVI could add a layer of biological information that will be valuable for cross-validating the results from the Human Impact Mapping effort. Essentially, the results of the EVI analysis can be compared with the human impacts map to check if some areas of ostensibly high value have been missed and should be further considered for management action. 
4. The approach must be iterative so that as each component is further developed or as data improves, the process can evolve and be conducted in a timeframe useful for management. While according to the Massachusetts Ocean Management Plan, the plan must be revised every five years, it may be appropriate to update the SSU maps more frequently. However, changes should be made carefully to ensure some consistency in management advice.

\subsection{Short/medium term}

Given the above recommendations, participants agreed to several objectives and tasks that can be accomplished in the short to medium term. Further development of habitat mapping, classification, and cumulative impacts maps needs to proceed as quickly as possible. This work is underway but should be considered a priority for funding. Cross-validation of the results of the EVI and the cumulative impact maps should be a relatively straightforward task once a base map is created by the human impacts mapping effort. This process would identify similarities and differences between the two approaches, lending insight into both the ecosystem-level vulnerability missed by the EVI and important species-level spatial features not captured by the cumulative impact model.

Scientists working on these approaches should consider available information on biodiversity, a component largely lacking in the existing approach. The Gulf of Maine Census for Marine Life may possess useful information. Participants also discussed the consideration of additional data sources into the approaches discussed above, including DMF trawl data, or more specifically, sub-dividing trawl data from aggregated data to match the spatial scale of other data sources. Temporal issues with the data (e.g. data taken at different times of the year) will still exist and will have to be addressed in the long term. Grouping species into guilds would also help inform the management plan in the short term. This action would allow the plan to achieve some parity in species groups without the need for weighting criteria in the EVI.

\subsection{Long term}

Habitats are relatively coarsely defined in the current approaches. Improving the resolution of the analysis may help improve the contrast between areas and distinguish those areas in need of greater protection. Another long term recommendation of the workshop participants is to consider a broader set of priority uses for different areas. The plan requires a defined set of uses, which currently does not include a use for environmental protection, conservation, or biodiversity. There are no existing rules for where it is safe to develop new activities (e.g. open ocean aquaculture); instead, all determinations are currently based on existing activities and current ecosystem characteristics. This leaves out the potential of recovery of habitats and resources. By expanding the definition of 'use' to include conservation, protection, or biodiversity, the plan can set aside areas for conservation or be used identify and protect areas of high biodiversity or those with potential as habitat for rebuilding of depleted stocks. Predictive models should be explored to understand which habitats species may reoccupy if rebuilding occurs or how ranges may change based on other influences (e.g. climate change).

The other tools considered in the workshop should not be neglected. It will be critically important to develop the capability to do trade-off analyses between different activities or services. The data 
developed for human impact mapping can be directly utilized in this effort. The trade-off analysis modeling effort should be supported as a major contribution to future management advice.

Similarly, for particular management needs, the PSA and HSI efforts can augment the broader scale analyses of multiple uses recommended above. A PSA analysis may be appropriate within a smaller region to highlight the most vulnerable fish stocks, for example, as part of a deeper analysis of the impacts that might occur in a particular area. HSI may be helpful as a means of evaluating habitat that might support recovering fish stocks or those areas that may be critical habitat under different climate scenarios.

Overall, the set of available approaches for mapping and comparing habitat will be powerful tools for advising managers implementing the Massachusetts Oceans Plan. This work needs to be supported and moved forward as quickly as possible. 